\title{
Der Weg ist es wert
}

\section{Anna Schaffelhuber}

Anna Schaffelhuber ist eine ehemalige Monoskibobfahrerin. In ibrer sportlichen Laufbabn nabm sie dreimal an den Paralympics teil und gewann dabei insgesamt sieben Goldmedaillen sowie je eine Silber- und Bronzemedaille. Die mehrfache Weltmeisterin und Gesamtweltcupsiegerin wurde 2020 vom Deutschen Behindertensportverband zur Para-Sportlerin des Jahrzehnts gekürt und arbeitet nach ihrem Rücktritt mittlerweile als Realschullehrerin.

Drei Paralympics-Teilnahmen, neun Medaillen, davon alleine fünfmal Gold in Sotschi 2014: In meiner Karriere habe ich mehr erreicht, als ich mehr das je hätte erträumen können. Ich durfte mein Land auf der großen Bühne der Paralympics präsentieren und kann noch heute meine Bekanntheit als Botschafterin meiner Sportart nutzen. Und auch wenn manche Aspekte der paralympischen Realität nicht immer den idealisierten Vorstellungen der Öffentlichkeit entsprechen, will ich diese Erfahrungen nicht missen.

Schon bei Olympischen Spielen ist der Unterschied in der medialen Wahrnehmung zu Welt- und Europameisterschaften enorm. Im paralympischen Sport ist das Gefälle noch extremer. Während einer normalen Saison fliegen wir da meist unter dem Radar, sodass die Paralympics für alle Athlet*innen eine enorme Bedeutung haben und eine wichtige Plattform darstellen, um den paralympischen Sport in die Gesellschaft zu tragen.

Dazu kommt, dass dort alle Wintersportdisziplinen vertreten sind. Viele Athlet*innen berichten von einem großen Gemeinschaftsgefühl auch über die eigene nationale Delegation hinaus. Leider konnte ich diesen völkerverbindenden Aspekt nie so ganz nachvollziehen. Für mich standen in den Tagen und Wochen rund um die Wettkämpfe zu viele sportliche und mediale Termine auf der Agenda, als dass für den Austausch mit anderen Nationen im paralympischen Dorf viel Zeit blieb.

Der Friedensgedanke war hingegen für mich unter den Sportler*innen gerade in Sotschi sehr präsent und gerade angesichts der damaligen KrimKrise sehr beeindruckend. Die russischen und ukrainischen Sportler*innen saßen gemeinsam beim Essen und tauschten sich völlig entspannt und normal miteinander aus. Die Ebene des gegenseitigen Umgangs war eine 
rein sportliche, die Politik spielte da nur für die Öffentlichkeit und in Interviewanfragen eine Rolle.

Das öffentliche Interesse an Äußerungen zu Themen abseits des Sports kann ich sehr gut nachvollziehen. Sportler*innen sind für viele Menschen Vorbilder, deren Aussagen aufgrund ihrer Popularität ein gewisses Gewicht haben. Wir können Themen eine Stimme geben, und ich habe diese Möglichkeit auch immer gerne für Themen genutzt, die mir am Herzen lagen. Es muss aber Grenzen geben, und mitunter habe ich mich da von Journalist"innen und Funktionär"innen auch etwas instrumentalisiert gefühlt. Gerade im direkten Umfeld der Wettkämpfe oder von Siegerehrungen muss die sportliche Leistung auch Thema genug sein.

Allgemein ist die Erwartungshaltung an Sportler*innen groß und für mich gerade im Leistungssport etwas zwiespältig. Sie sollen immer das Maximum ihrer Leistungsfähigkeit abrufen und möglichst viele Medaillen holen, dabei aber immer auch ein einwandfreies Verhalten zeigen und so Vorbild für Kinder und Jugendliche sein. Gerade als Pädagogin kann ich diesen Wunsch nachvollziehen, denn vorbildliches Verhalten strahlt ganz besonders auch auf die untersten Leistungsebenen und Altersklassen positiv ab. Die ehemalige Leistungssportlerin in mir sieht da aber auch die andere Seite. Denn um auf höchstem Level zu bestehen, benötigt es auch ein gewisses Maß an Egoismus. Gerade ein Wert wie Freundschaft stößt da unter direkten Konkurrent*innen durchaus an Grenzen und hat sich auch in meiner Karriere nie ganz verwirklichen lassen.

Trotz der Einschränkungen im Spitzensport würde ich die Relevanz der olympischen Werte immer wieder unterschreiben. Gerade im Breitensport- und Nachwuchsbereich liefern sie unglaublich wertvolle pädagogische Anknüpfungspunkte und können viel stärker mit Leben gefüllt werden, als das an der Spitze vielleicht der Fall ist. Und selbst dort konnte ich in Einzelsituationen feststellen, wie fest ein Wert wie Respekt auch auf höchstem Niveau verankert sein kann: In Sotschi etwa, als ich wegen eines vermeintlichen Startfehlers disqualifiziert wurde, setzten sich andere Nationen dafür ein, dass mein Lauf doch gewertet wurde. Für mich war dieser Respekt unter Konkurrent*innen fast so wertvoll wie die Goldmedaille.

Neben diesen großen Idealen sind es aber gerade auch die vermeintlichen Nebeneffekte einer leistungssportlichen Karriere, aus denen ich viel für mein Leben ziehen konnte. Der positive Umgang mit Niederlagen, aus denen ich immer viel mitnehmen konnte, hat mich sicherlich geprägt. Dazu musste ich schon in jungen Jahren lernen, meinen Alltag gut zu strukturieren und diszipliniert verschiedene Aufgaben zu bewältigen. Ich habe nie alles auf eine Karte gesetzt und mir zum Glück in 
der Schule immer leichtgetan, sodass ich mir neben dem Sport etwas aufbauen konnte. Auch im Training selber konnte ich mich dank meiner Trainer*innen schon früh selbst einbringen, Dinge ausprobieren und den Prozess mitgestalten. Für meine Motivation war das ein wichtiger Faktor.

In meinem neuen Leben als Lehrerin kann ich diese Entwicklungen nun auch an einzelnen Schüler*innen beobachten, die sich in Richtung Leistungssport orientieren. Schon nach wenigen Wochen waren dabei deutliche Auswirkungen zu spüren: Sie hatten mehr Struktur gelernt und Werte wie Fair Play, Respekt und Teamplay wesentlich stärker verinnerlicht als zuvor. Leistungssport ist sicherlich kein Wundermittel, das möchte ich damit nicht ausdrücken. Wenn allerdings Kinder den Wunsch haben, es zu versuchen, würde ich sie immer dabei unterstützen. Auch wenn sie nicht ganz oben ankommen, können sie auf diesem Weg viel Positives mitnehmen.

Im Jahr 2019 habe ich meine aktive Karriere beendet und genieße es jetzt, ohne Leistungsdruck sportlich aktiv zu sein - auch wenn mitunter der Ehrgeiz immer noch die Oberhand gewinnt. Vielleicht gelingt es mir ja jetzt als Athletin im Ruhestand, auch den Aspekt der Völkerverständigung bei den kommenden Paralympics noch mehr aufzusaugen, als mir das in meiner aktiven Laufbahn gelungen ist. Denn unter den Zuschauer*innen, so wurde mir von meiner Familie gespiegelt, wurde der Gedanke der Völkerverständigung komplett gelebt. Gerade 2026, wenn die Paralympics nach Cortina d'Ampezzo und damit endlich wieder in eine traditionelle Wintersportregion kommen, wird es daher sicherlich ein tolles Erlebnis sein, die Wettkämpfe aus einer anderen Perspektive zu verfolgen. 
